\title{
Simulation Analysis of Non-stationary Random Noise Modeling in an Automobile Engine environment
}

\author{
S. Thilagam ${ }^{1}$ and P. Karthigaikumar ${ }^{2}$ \\ ${ }^{1}$ Department of Electronics and Communication Engineering \\ Kumaraguru College of Technology \\ Coimbatore - 641049 \\ ${ }^{2}$ Department of Electronics and Communication Engineering \\ Karpagam College of Engineering \\ Coimbatore - 641032 \\ 1'thilagamsundar@gmail.com, ${ }^{2}$ p.karthigaikumar@gmail.com
}

\begin{abstract}
Various types of non-stationary noises that occur in a car environment are due to engine, belts, tires and braking systems. The interior noise in a car cabin includes tire noise, Road Noise, wind Noise and engine noise. These noises exist at low frequencies and can be cancelled using passive and active noise control techniques. Passive noise control techniques uses insulation materials and mufflers at the cost of increased weight. Active noise control methods in present day cars uses digital signal processing tools to analyze the various real-time noises and cancels them providing a smooth riding experience to the user. The problem of cancelling these non-stationary Interior noises in an automobile car environment requires thorough study of various categories of noises and analysis of those noise signals in terms of their probability density functions and power spectrum. This paper provides detailed analysis of various noises present in a Real-time automobile cabin environment with their spectrum analysis using proven results.
\end{abstract}

Keywords: Automobile Engine Noise, Random Noise Modeling, Active Noise Control, Digital Signal Processing

\section{Introduction}

Noise is an unwanted signal which interferes during communication or in the measurement of an information signal. Noise is present in various levels in almost all environments. The noises in a car environment arise due the engine, belts, tires and braking systems. Many noise processing techniques are involved to characterize and model the noise process [1,2].Noise inside a car occurs due to interior noises and exterior noises. Interior Noise is the source of Noise in IC engine which is due to the piston crank mechanism and combustion process. This interior noise also occurs due to road noise from varied road surfaces and tire noise and braking mechanism. All these noises enter the cabin causing discomfort to the passenger. These noises have to be controlled and minimized to have a smoother riding experience to the passenger.

This paper provides an extensive analysis of Real-time noise signal in a car environment and presents a suitable active noise cancellation technique for an automobile in running condition. This paper is organized as follows. Section 2 discusses the various types of Automobile noises, Section 3 illustrates the characteristics of the noise signal in a car environment, Section 4 provides various probability models of the Vehicle Interior Real-time noises, Section 5 gives the spectral analysis of interior noise of a running car 
and simulation of these noises are done using MATLAB tool box and the results are discussed in Section 6. Conclusion is presented in Section 7.

\section{Types of Automobile Noise}

A noise can be classified into a number of categories depending on the sources that produce them.

Any noise generated from moving, vibrating or colliding sources highly found in moving cars is termed as Acoustic Noise. Another type of noise called Thermal Noise and Shot Noise is created by the random movement of thermally energized particles in an electric conductor. Shot Noise consists of random fluctuations of the electric current in an electrical conductor $[2,3]$. The presence of a voltage with or without current flow in a system induces Electromagnetic noise. A purely random noise that has a flat power spectrum is termed as White Noise. It is defined as an uncorrelated random noise process with equal power at all frequencies [4-6].These noises are studied and analyzed using digital signal processing techniques.

The autocorrelation function of a continuous-time zero-mean white noise process with a variance $\sigma^{2}$ is a delta function $[7,8]$. The illustration of white noise with zero mean and standard deviation of four is shown in Figure 1.The theoretical probability density function for a white Gaussian noise is calculated and its simulated histogram is observed .It is shown in Figure 2.

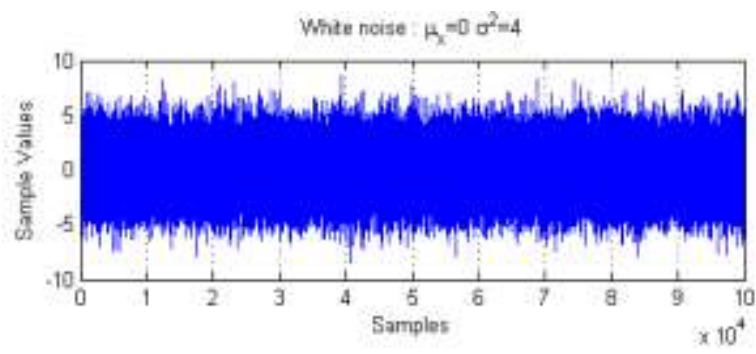

Figure 1. White Noise with Zero Mean and SD=4

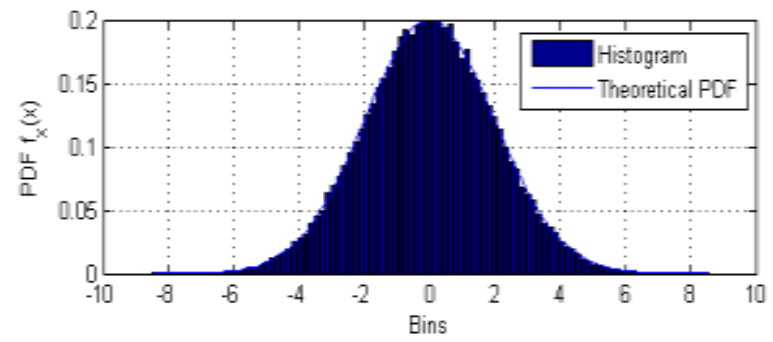

Figure 2. Theoretical PDF and Histogram of White Gaussian Noise

Many noises that consist of non-white spectrum are termed as Colored Noises. Noise from moving cars has a non-white predominantly low-frequency spectrum $[9,10]$. Also, a white noise passing through a channel is colored by the shape of the frequency response of the channel. Two classic type of colored noise are Pink noise and Brown Noise. The Electromagnetic noise comes from man-made devices or Natural sources. The main source of electromagnetic noise in an automobile comes from electric motors and electric drives. The advanced hybrid electric vehicle technology used in cars increases the electromagnetic and thermal noises. 


\section{Noise Characteristics in a Car Environment}

The objective of modeling is to characterize the structures and the patterns in a signal or noise process. The noise modeling is achieved by proper noise statistics. The use of Fourier transform for frequency analysis is an indispensable tool for noise modeling. The analysis of Noise characteristics that arise inside the car cabin provides a suitable strategy to control and minimize them.

The noise in a Car environment is non-stationary and varied including quasi-periodic noise from the car engine and revolving mechanical parts of the car. A noise in a car environment often occurs due to surface contact of wheels and road surface, noise from air flow into the car through air ducts, windows, noise from engine, throttle. The characteristics of car noise vary with speed, road surface conditions, weather and environments within the car. The noise modeling is done to estimate the noise statistics from the signal-inactive periods [11]. The noise that occurs in a real-time car environment is time-varying, correlated and non-Gaussian as shown in Figure 3.

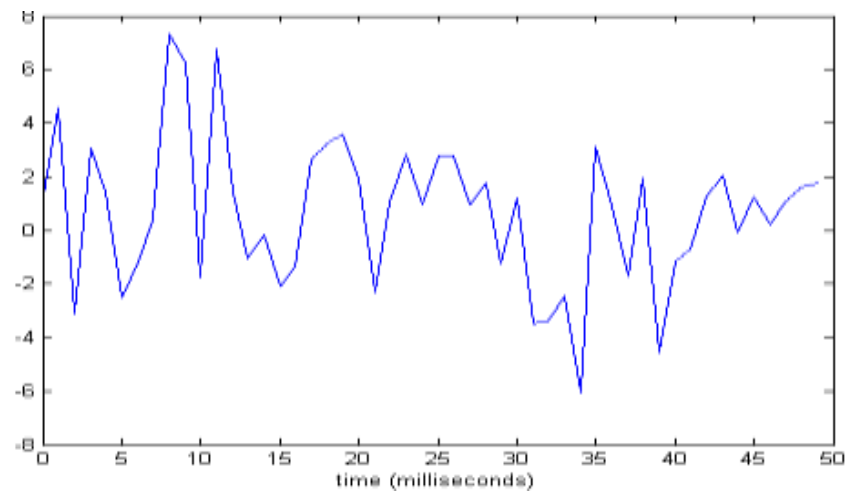

Figure 3. Random Engine Noise signal

Signals are classified into two types namely Deterministic signals and Random Signals. Deterministic signals, for example Sine wave that has no information content on their own but modulated by a random information-bearing signal. Random Signals, for example Speech, image that have information.

A deterministic signal has a predefined trajectory in time and space. The exact value at any time is predictable from the functional description and the past history of the signal. Random signals have unpredictable fluctuations. Therefore it is possible to predict the exact future value of a random signal. Although, a random signal cannot be predictable, it provides a set of well-defined statistical values such as maximum and minimum mean, median, variance, power spectrum and higher order statistics. The future values of Random signals that occur in real-time environment are not predictable from the past values [12]. The statistics and probability theory forms the basis for modeling the randomness in a signal. A Random process is characterized with statistics ie. Mean, correlation and the power spectrum. The effect of filtering or transformation of a signal on its probability distribution is studied using random process.

\section{Modeling of Vehicle Interior Noise}

A car's engine produces various ranges of frequencies including the dominant frequency. These vibrations are derived from the crankshaft cylinder and the combustion process. There are various methods to identify and model the vehicular noise sources. The most basic method is the measurement of noise in Near-field. The frequency spectrum analysis and Coherent analysis are done for finding the peak value frequency of noise 
power to identify the major source. The Block diagram of the Noise identification and measurement system is shown in Figure 4.

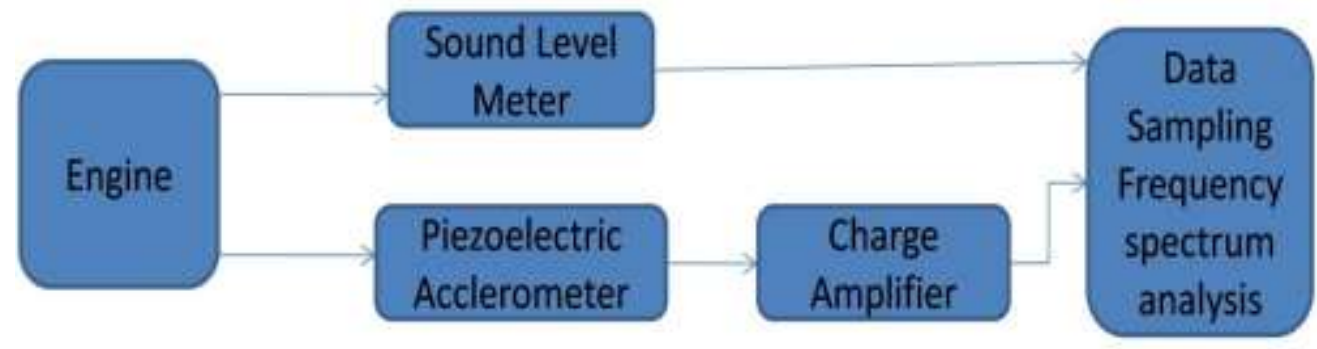

Figure 4. Block Diagram of Engine Noise Measurement System in a Car

The sound level meter is used to capture the engine noise level which is further controlled and minimized. The frequency spectrum of the engine noise can be found by analyzing the engine noise observed from Sound Level meter. The Noise captured from the meter includes noise from airflow vibration and intake pipe during engine running condition. The frequency analysis of the engine noise measured using sound level meter is done using the Discrete Fourier Transform of digital signal processing technique.

Various sensors are used to capture the noise inside the interior of the car especially using suitable microphones. The Active noise control techniques to capture the noise signal include the use of Microphones and Loud speakers. The engine exhaust noise thus obtained is different for no-load and full- load conditions. The noise level is usually $10 \mathrm{~dB}$ higher in full load condition compared to no-load condition. The Noise measured inside the car is done using a Feed forward control system or a Feedback control system. The feed forward control system uses a reference signal which is correlated with the existing interior noise signal for effective cancellation of noise. The feedback system does not have a reference signal as it is very difficult to find a reference signal for tire noise and Road booming noise.

The Feed forward control system is thus used to capture the interior signal inside the car cabin using the Microphone and loudspeakers and is fed to the Adaptive filter for minimizing them. The system with initial set up can be a single input and single output system minimum microphones and loudspeakers. It can be extended to Multiple inputs multiple output feed forward system using four or six microphones and loudspeakers. The Interior noise measurement and cancellation system using Feed forward control method is shown in Figure 5.

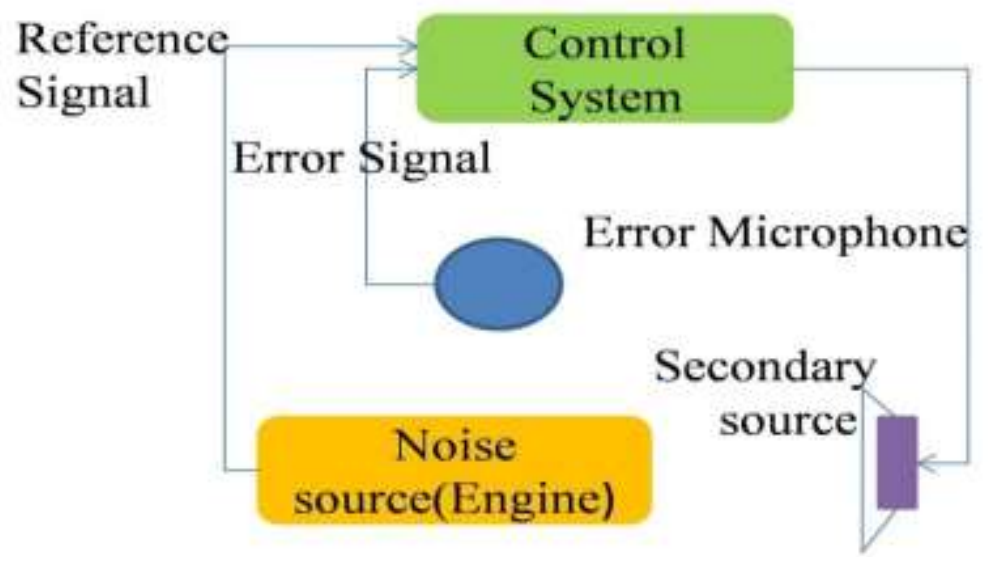

Figure 5. Feed Forward Noise Control Mechanism 
Probability models utilize the estimation of the likely values of a process from noisy or incomplete observations. Probability models are the functions of statistical parameters of Random process ie.exponential functions of the mean value and covariance of the process. The probability of a discrete-valued random variable is called the Probability mass function (pmf).and the continuous-valued random variable is called Probability Density function (pdf).A Histogram is a graph that shows the number of times the value of a random variable occurs in each uniform interval in a given set of observations of the variables. The Gaussian and Uniformly distributed Random Signal and their Probability Density functions of engine noise occurring in a car cabin are illustrated in Figure 6.

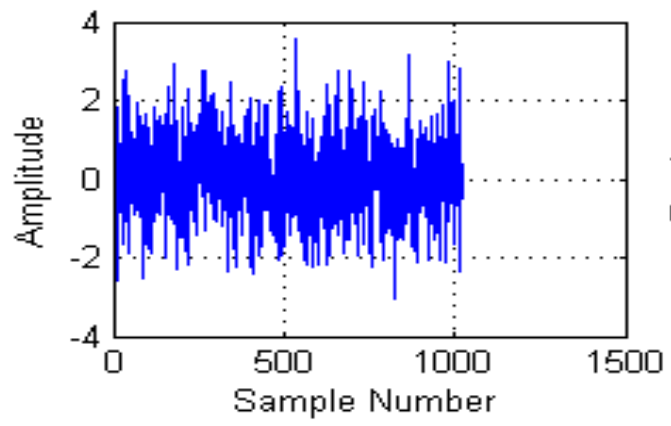

a) Normal Distribution of Non-stationary Engine Noise Signal

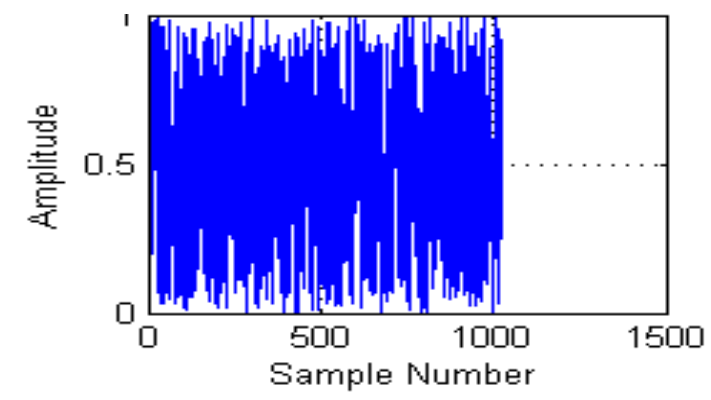

c) Uniformly Distributed Non-stationary Engine Noise Signal

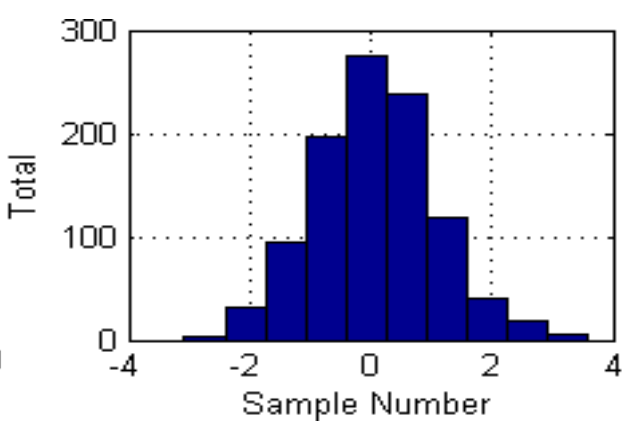

b) Histogram (PDF) of Non-stationary Engine Noise Signal

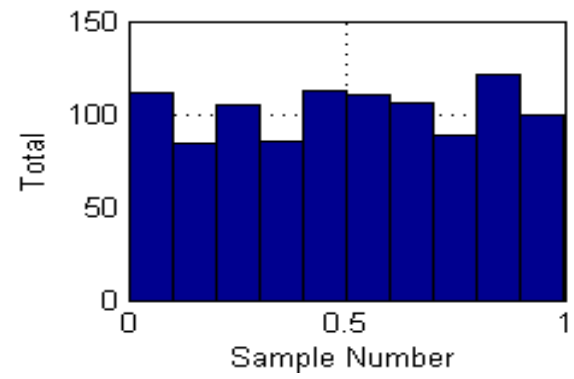

d) Histogram (PDF) of Non-stationary Engine Noise Signal

Figure 6. Interior Noise Signal and Their PDF

\section{Spectral Analysis of Vehicle Interior Noise}

The process that generates the noise signal may be time invariant(stationary) or time varying(non-stationary).The statistical parameters such as mean, variance, the power spectral composition and higher-order moments of the process are constant for a time invariant signal where as a random process may have a time-invariant mean but a timevarying power. The two models of a non-stationary process are static mode and timevarying signal mode.

A stationary process drives the parameters of continuously time-varying models. A finite-state model with each state having a different set of statistics. The expected values of a random process, also known as its statistics are the mean, variance, correlation, power spectrum and the higher-order statistics of the process. The most important expected values are the first-order moment ie. Mean value and the second-order moments namely the correlation, the covariance and the power spectrum $[13,14]$. 
The estimate of the mean $\mu_{\mathrm{x}}$ from a finite number of samples of a signal $\mathrm{x}(\mathrm{m})$ is calculated[15,16]. The autocorrelation of a random process $\mathrm{X}(\mathrm{m})$, denoted by $R_{x x}(m 1, m 2)$ is ,

$$
R_{x x}(m 1, m 2)=E[(x(m 1) x(m 2)]
$$

The autocorrelation function is a measure of the similarity or the mutual relation of the outcomes of the process $\mathrm{X}$ at time instants $\mathrm{m} 1$ and $\mathrm{m} 2$.The auto covariance function $C_{x x}(m 1, m 2)$

of a random process $\mathrm{X}(\mathrm{m})$ is a measure of the scatter or the dispersion of the process about the mean value and is defined as

$$
C_{x x}(m 1, m 2)=r_{x x}(m 1, m 2)-\boldsymbol{\mu}_{x}(m 1) \boldsymbol{\mu}_{x}(m 2)
$$

The autocorrelation function of a white noise is shown in Figure 7.

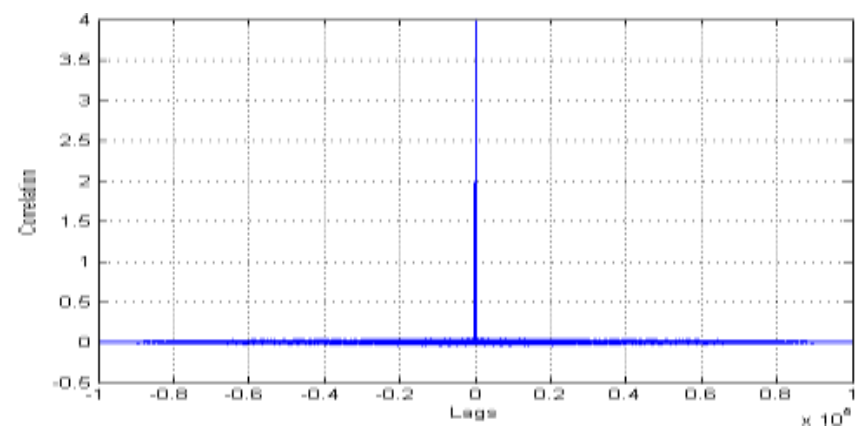

Figure 7. Autocorrelation Function of Automobile Engine Noise

The power spectral density function $\mathrm{n}$ also called power spectrum of a process gives the spectrum of the distribution of power at different frequency of vibrations along the frequency axis.

$$
\begin{aligned}
& p_{x x}(f)=E[x(f) x *(f) \\
& p_{x x}(f)=\sum r_{x x}(k) e^{-j 2 \pi f n}
\end{aligned}
$$

The Power Spectral Density Function is a real-valued non-negative function expressed in units of watts per hertz. The spectrum of a random noise signal of engine noise in an automobile is shown in Figure 8.

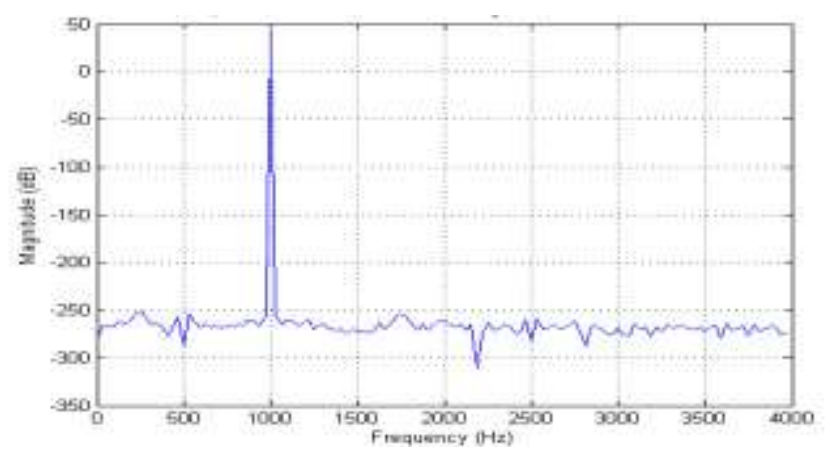

Figure 8. Spectrum of Non-Stationary Engine Noise Signal 


\section{Simulation Results and Discussion}

The analysis of these non-stationary engine noises are illustrated by simulation results using MATLAB. The power spectrum of Random noise of an engine in an automobile environment is illustrated in Figure 9. For many signal processing problems, the process of getting an output of an array of sensors is done by using more than one random process. Joint statistics and joint distributions are used to describe the statistical interrelationship between two or more random process. The Gaussian process also called the normal process is the most widely applied probability models to model noise. The Random signals obtained at various time intervals in a car engine are illustrated in Figure 10 .

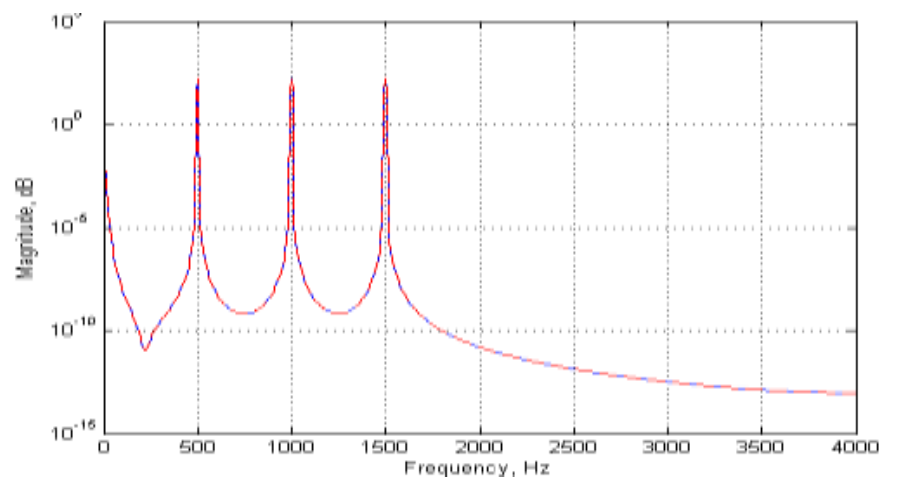

Figure 9. Power Spectrum of a Vehicle Engine Noise
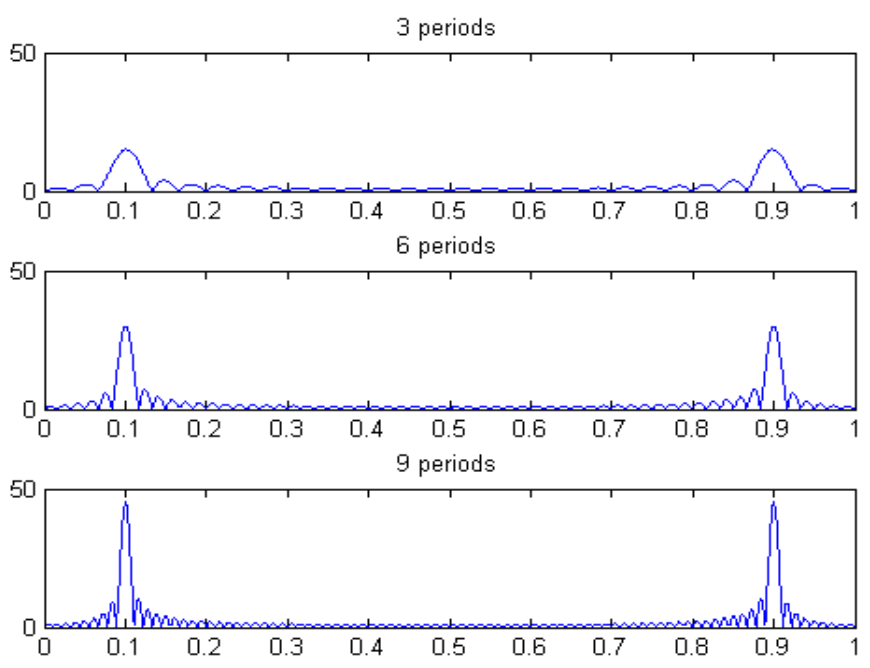

\section{Figure 10. Random Noise Signals in a Car engine Environment in Different Time Intervals}

It is inferred from Figure 8 that as time interval increases, the random noises tend to increase thereby increase the complexity of cancelling them. The use of Active noise control strategies using Adaptive filter techniques provides best alternatives to passive mufflers in such noise cancellation. The above simulation results describe the characteristics and modeling of the low frequency Engine noise. This can be extended to analyze other noises like Tire noise, Road noise and wind noise that contributes to the overall interior noise inside the car cabin. Once the characteristics of the Interior noise 
signal are known, it can be effectively minimized using the Active Noise Control techniques using adaptive filter techniques.

\section{Conclusion}

The modeling of Interior noise signals from Engine due to crank shaft mechanism and combustion process and its analysis in a real-time car environment is presented with MATLAB simulation results. This analysis helps in providing better Noise Cancellation strategies in a non-stationary environment. Passive Noise cancellation methods like using insulation materials and mufflers can thus be replaced by active noise control methods and enables effective manufacturing of light weight cars. Active noise control methods using Adaptive filtering is necessary to cancel out these noises since it occurs in real-time to provide better riding experience to the user.

\section{References}

[1] S. Gharavi, "A new, delay-line-discriminator based, hybrid RF/digital phase noise Cancellation technique, Circuits and systems", (ISCAS), IEEE International Symposium, (2013).

[2] G. Davis, "Noise reduction in speech applications", Electrical engineering and applied signal Processing series, CRC press publication, (2002).

[3] V.G. Galushko, "Analysis of the CA CFAR algorithm as applied to detection of stationary Gaussian signals against a normal noise background", 9th International Kharkiv Symposium on Physics and Engineering of Microwaves, Millimeter and Sub millimeter Waves (MSMW), (2016).

[4] S.V. Vaseghi, "Advanced Digital Signal processing and Noise Reduction- $2^{\text {nd }}$ Edition", John Wiley \& sons publication Ltd, (2000).

[5] C. Osterwise, "Reduction of noise in near-field measurements", Electromagnetic Compatibility (EMC), IEEE International Symposium, (2010).

[6] J.E. Paul, "Adaptive digital techniques for audio noise cancellation", Circuits and systems Magazine, California State.

[7] C. Desset, "Signal, noise and interference power analysis in MRT-based Massive MIMO systems", IEEE International Symposium on Circuits and Systems (ISCAS), (2016).

[8] D. Petrinovic, "Digital Signal Processing", ZESOI, FER, University of Zagreb, (2002).

[9] M. Sen, "Design of active noise control systems with the TMS320 family".

[10] K. Kim, "A new approach for Rustle noise canceling in pen-type voice Recorder", Consumer Electronics, IEEE Transactions, (2003).

[11] N. Ascendorf and R.R. Nadakuditi, "Improved detection of correlated signals in low-rank- plus-noise type datasets using Informative Canonical Correlation Analysis", IEEE transactions on Information Theory, (2017).

[12] K.T. Andersen, M. Moonen, "Adaptive time frequency analysis for noise reduction in an audio filter bank with low dela", IEEE Transactions on audio, speech and language processing, vol. 24, no. 4, (2016), pp. 784-795.

[13] S. Thilagam and P. Karthigaikumar, "Implementation of adaptive noise canceller using FPGA for realtime applications", Electronics and Communication Systems (ICECS), 2015 2nd International Conference on. IEEE, (2015).

[14] R. Martin, "Noise power spectral Density estimation based on Optimal smoothing and minimum statistics", IEEE transactions on speech and audio processing, vol. 9, no. 5, (2001).

[15] N.P. Narendra and K.S. Rao, "A deterministic plus noise model of excitation signal using principal component analysis for parametric speech synthesis", IEEE International Conference on Acoustics, Speech and Signal Processing (ICASSP), (2016).

[16] S. Thilagam and P. Karthigaikumar, "Design of An Adaptive Noise Cancellation System in A Time Varying, Non-Linear Automobile Environment”, i-Manager's Journal on Digital Signal Processing, (2016). 


\section{Authors}

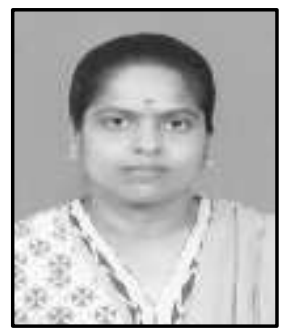

S. Thilagam, she is working as an Assistant Professor in Department of Electronics and Communication Engineering, Kumaraguru College of Technology, Coimbatore. She has academic and teaching experience of over ten years. She completed her M.E. in VLSI Design and is currently pursuing PhD in Anna University, Chennai. She has published papers in several national and international conferences and journals. Her area of interest includes VLSI Signal Processing, Automotive Electronics and Embedded Systems.

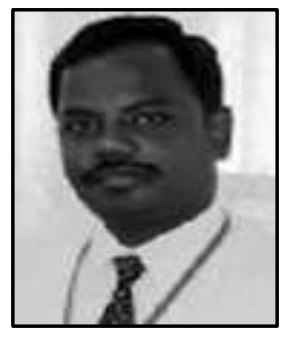

P. Karthigaikumar, he is working as a Professor in Department of Electronics and Communication Engineering, Karpagam College of Engineering, Coimbatore. He had completed his Doctorate in Anna University, Chennai and has more than fifteen years of academic and teaching experience. He has published many papers in international conferences and journals. He has also filed two patents. His area of interests includes VLSI Design and Cryptography. 
International Journal of Control and Automation

Vol. 10, No. 10 (2017) 